\title{
DIFFUSION IN CP STARS: THE QUEST FOR ACCURACY
}

\author{
G. ALECIAN \\ Observatoire de Paris \\ DAEC, Observatoire de Meudon, F-92195 Meudon Cedex, FRANCE
}

\section{Introduction}

We present a brief review about recent progresses concerning the study of diffusion processes in CP stars. The most spectacular of them concerns the calculation of radiative accelerations in stellar envelopes for which an accuracy better than $30 \%$ can now be reached for a large number of ions. This improvement is mainly due to huge and accurate atomic and opacity data bases available since the beginning of the 90's. Developments of efficient computational methods have been carried out to take advantage of these new data. These progresses have, in turn, led to a better understanding of how the element stratification is building up with time. A computation of self-consistent stellar evolution models, including time-dependent diffusion, can now be within the scope of the next few years. However, the progresses previously mentioned do not apply for stellar atmospheres and upper layers of envelopes.

\section{Recent progresses}

\subsection{MAIN STEPS}

Before to present some recent progresses, it would be helpfull to briefly recall what are the main steps in diffusion computations in CP stars. Firstly, one has to compute the radiative accelerations on ions. For this, one needs (i) atomic data for considered ions, (ii) stellar model (atmosphere and/or envelope, and (iii) one needs to calculate the photons flux at each wavelength and at each depth. Secondly, one needs to compute the diffusion velocities which depend strongly on the radiative accelerations and gravity. The final step is the solving of the time-dependent continuity equation in order to find how element stratifications proceed.

\subsection{THE RADIATIVE ACCELERATION}

The average acceleration on an ion $\mathrm{A}^{+i}$ due to bound-bound (bb) transitions $l$ (hereafter, we assume that photoionization has negligible contribution) is:

$$
g_{i}^{r a d}=\sum_{l} \frac{1}{A_{i} m_{p} n_{i}} \int_{0}^{\infty} n_{i k} \sigma_{i, k m}(\nu) \frac{\phi(\nu)}{c} d \nu
$$

Where $\phi(\nu)$ is the net photons energy flux $\left(\mathrm{erg} \cdot \mathrm{cm}^{-2} \cdot \mathrm{s}^{-1}\right), \mathrm{n}_{i k}$ is the number of ions $\mathrm{A}^{+i}$ (with mass $A_{i} m_{p}$ ) in level k per $\mathrm{cm}^{3}, \sigma_{i, k m}$ absorption cross-section for $l$ (from level $\mathrm{k}$ to $\mathrm{m}$ ). To have a good estimate of $\mathrm{g}_{i}^{r a d}$ one needs atomic data for all lines and not only for the observed ones. Another potential difficulty is that the flux $\phi(\nu)$ is non-local in atmospheres (optically thin). However, it is local and easy to compute in stellar envelopes (optically thick).

\subsubsection{New data and new methods}

Presently, the availability of huge atomic and opacity data bases (TOPbase, OPAL) has two consequences: (i) to improve the accuracy of the radiative accelerations, (ii) to reduce the number of missing ions. But, the amount of transitions $l$ in computations of $\mathrm{g}_{i}^{\text {rad }}$, consists in thousands of lines that make the calculations very heavy, since the radiative acceleration must be computed each time 
the local concentration of A changes. To overcome this problem and to take advantage of these new data, several techniques have been developed. They apply to optically thick cases.

One kind of method could be named as "detailed \& accurate". Two of this kind have been recently developed: the one by Richer et al (1997), and the one by Seaton (1997). Both methods, which present some similarities, are based on extensive use of OPAL or OP data. Richer et al (1997) compute the integral over frequencies in $g_{i}^{r a d}$ from opacity tables provided by OPAL. The detailed atomic data are not explicitly needed since lines effects are included in the detailed opacity tables for each element. The total monochromatic opacity and the Rosseland average are computed each time local concentrations of elements are changed and therefore, this method is well adapted for complete and self-consistent computations. Seaton (1997) computes the same integral from tables provided by $\mathrm{OP}$, and uses the detailed opacity tables for each ion. These accelerations are accurate but only one element at a time is allowed to diffuse. Results are stored at CDS (Strasbourg).

Another kind of method is the " $\phi \& \psi$ " approximation (Alecian \& Artru, 1990). Here, Lorentz profiles are assumed for lines and, photoionization effects on accelerations are neglected. The background monochromatic opacity is supposed to be equal to the Rosseland opacity (in average). Then, $\mathrm{g}_{i}^{\text {rad }}$ takes the very simple following form $\left(C_{i}\right.$ is the local ion concentration):

$$
g_{i}^{r a d} \approx q \phi_{i}\left(1+\frac{C_{i}}{b \psi_{i}^{2}}\right)^{-\frac{1}{2}}
$$

The functions $\phi_{i}$ and $\psi_{i}$ depend mostly on the local temperature and atomic data, while $q$ and $b$ depend on the stellar model and depth. The main interest of this method is that $\phi_{i}$ and $\psi_{i}$ could be tabulated independently of the stellar model and of $C_{i}$. Its accuracy is good for test particles, provided that the $\phi_{i}$ and $\psi_{i}$ were calibrated using detailed computations (Alecian, Michaud, Tully, 1993). An improvement of this method is planned in the short term.

\subsection{STRATIFICATION PROCESSES 1.3.1.}

\subsubsection{Time-dependent diffusion (optically thick cases)}

Element stratification is a time-dependent process and obeys to the following continuity equation, where $n$ is the element number density, $V_{i}$ the diffusion velocity of ion $i, V_{M}$ a macroscopic flow velocity:

$$
\partial_{t} n+\nabla_{r}\left(\sum_{i} n_{i} V_{i}+n V_{M}\right)=0
$$

This equation is very difficult to solve because of strong non-linear terms in $V_{i}$. On another hand, the time scales involved in the process are sometimes of the same order of magnitude as the time scales of stellar evolution on the main sequence. Alecian (1996) solves this equation numerically. He keeps all the non-linearities, but uses the " $\phi \& \psi$ " approximation for $\mathrm{g}_{i}^{r a d}$. His method is only valid for test particles, and stellar evolution is neglected. Seaton (1996) solves the continuity equation in peculiar cases and assumes $V_{M}=0$ : under some assumptions (see Seaton, this conference) the continuity equation may be linearized and reduces to a wave equation for fluxes. This method cannot be generalized to any element and to any star. Turcotte et al. (1997) solve the full continuity equation (see Michaud, this conference). The diffusion velocities (using the "detailed \& accurate" method) of many elements are computed simultaneously in self-consistent models. Presently, they consider the case $V_{M}=0$, but this method appears to be the most promising one to solve the time-dependent diffusion problems. It is, however, very heavy to carry out.

\subsubsection{What about the Ap stars atmospheres?}

The time-dependent diffusion in stellar atmospheres is much more difficult to study than in envelopes. Since the medium is optically thin, the continuity equation is coupled to the equation of radiation transfer. The various approximations used for optically thick cases no more apply. The expected computation time should be much greater compared to the previous case. Moreover, new specific numerical difficulties should be expected. As far as we know, there is still no attempt to solve these coupled equations.

However, interesting results have been obtained with a "zero order " approximation by solving: 


$$
g^{\text {rad }}\left(n_{\text {equilib }}\right)=g
$$

In this expression $n=n_{\text {equilib }}$ is a solution such as diffusion velocity is close to zero everywhere (see for instance Alecian \& Michaud 1981). A better solution can be obtained by using an equation imposing constant flux of element (steady state solution $n_{s t}$, see Babel, 1992). However several problems arise: (i) the upper boundary conditions are badly known, (ii) there is no evidence that $n_{\text {equilib }}$ (or $n_{s t}$ ) is always solution of the continuity equation coupled to the equation of transfer. On another hand, element stratifications may be unstable (see Alecian \& Grappin 1984, Alecian, 1997).

\section{Observations of Open clusters}

Open clusters are very interesting laboratories to study stratification processes in Am stars. They offer homogeneous samples of stars (in age and composition), the age of the stars could be known with a relatively good accuracy, it is possible to select young objects.

High resolution spectroscopy is needed to determine accurate metal abundance and several works have been done in that field [see for instance, Burkhart \& Coupry $(1989,1997)$, Hui Bon Hoa et al (1997), Hui Bon Hoa \& Alecian (1997)]. According to the available data, it seems that young Am phases are detected in young open clusters. Further detailed observations of open clusters will complete the statistics which are still very poor. This will increase significantly our knowledge on how stratification processes occur, but also on hydrodynamics in outer layers of A stars.

\section{Conclusion}

One can conclude that, presently, very accurate (better than 10\%) accelerations can be achieved for many elements and the time-dependent stratification processes can be carried out in the optically thick case. On another hand, some detailed abundances of A stars in open clusters become available and help to a better understanding of stratification processes.

For the future, clearly more atomic data are needed (specially for heavy elements). Very important progresses will be expected from self-consistent modeling of stars (including diffusion), the group of Montreal as already started to work in that field. This does not still concern the stratification processes in atmospheres, for which studies are planned in the short term. Concerning, observations, high resolution spectroscopic surveys of A stars in open clusters should give interesting insight about hydrodynamics involved in stratification processes. This should be possible in a few years through multiobject spectroscopy on VLT.

\section{References}

Alecian, G.(1996) Astron. Astrophys., Vol. 310, pp. 872

Alecian, G.(1997), in preparation

Alecian, G., Artru, M.C.(1990) Astron. Astrophys., Vol. 234, pp. 323

Alecian, G., Grappin, R.(1984) Astron. Astrophys., Vol. 140, pp. 159

Alecian, G., Michaud, G.(1981) Astrophys.J., Vol. 245, pp. 226

Alecian, G., Michaud, G., Tully, J.(1993) Astrophys.J., Vol.411, pp. 882

Babel, J.(1992) Astron. Astrophys., Vol.258) P.449

Burkhart, C., Coupry, M.F.(1989) Astron. Astrophys., Vol.220, pp. 197

Hui Bon Hoa, A., Alecian, G.(1997) submitted

Hui Bon Hoa, A., Burkhart, C., Alecian, G.(1997) Astron. Astrophys., Vol.232, pp. 901

Michaud, G., Richer, J.(1996) 13th Symposium On Spectral Lines Shapes (Florence)

Seaton, M.J.(1996) Physica Scripta, Vol.T65, pp. 129

Seaton, M.J.(1997) MNRAS, in press

Turcotte, S., Richer, J., Michaud, G.(1997) in preparation 